\title{
Comparison Between Primary and Secondary Method of Closing Surgical Wound After Tooth Extraction: A Split-Mouth Study
}

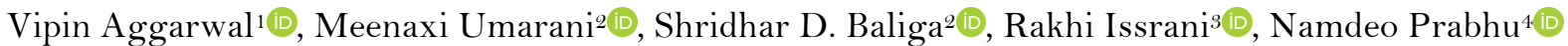

'Department of Oral and Maxillofacial Surgery, Kalka Dental College, Meerut, India.

'Department of Oral and Maxillofacial Surgery, V. K. Institute of Dental Sciences, KLE University, Belgaum, Karnataka, India.

${ }^{3}$ Department of Preventive Dentistry, College of Dentistry, Jouf University, Sakaka, Kingdom of Saudi Arabia.

${ }^{4}$ Department of Oral \& Maxillofacial Surgery and Diagnostic Sciences, College of Dentistry, Jouf University, Sakaka, Kingdom of Saudi Arabia.

Correspondence: Dr. Rakhi Issrani, Lecturer, Department of Preventive Dentistry, College of Dentistry, Jouf University, Sakaka, Kingdom of Saudi Arabia. E-mail: dr.rakhi.issranioo@gmail.com

Academic Editor: Catarina Ribeiro Barros de Alencar

Received: 22 October 2020 / Review: 09 December 2020 / Accepted: 28 December 2020 How to cite: Aggarwal V, Umarani M, Baliga SD, Issrani R, Prabhu N. Comparison between primary and secondary
method of closing surgical wound after tooth extraction: a split-mouth study. Pesqui Bras Odontopediatria Clín Integr.
2021; 21:e0227. https://doi.org/10.1590/pboci.2021.073

\begin{abstract}
Objective: To compare and assess the primary and secondary closure techniques following extraction of impacted third molars for post-operative complications. Material and Methods: In total, 30 patients ranging between 18-30 years of age and of either sex who had bilaterally impacted mandibular third molars were randomly selected. Split mouth study method was used so that the participants served as their own control. Group 1 consisted of primary closure of left mandibular impacted third molars and Group 2 consisted of secondary closure of right mandibular impacted third molars. Basement evaluations were recorded for each patient along with subjective and objective evaluations for postoperative 7 days. Data analysis was carried out by SPSS 17.0 software using Mann-Whitney U test, Wilcoxon matched-pairs test and t-test. A p-value $\leq 0.05$ was assigned as statistically significant. Results: When compared to group 1 , group 2 revealed statistically less pain and swelling following the secondary closure of wound from day 1 to 7. There was a significant improvement in mouth opening in Group 2 at day $1(p=0.0005)$ and at day 7 $(p=0.00001)$. Conclusion: Secondary wound closure after disimpaction of mandibular third molar results in better postoperative recovery than primary closure.
\end{abstract}

Keywords: Surgery, Oral; Pain, Postoperative; Suture Techniques; Trismus. 


\section{Introduction}

One of the most common surgical interventions in dental practice is an extraction of mandibular impacted third molars. As with any other surgical procedure, this procedure is also related with some postoperative sequelae, to name a few like pain, swelling, trismus, etc. [1]. These sequelae reflect inflammatory tissue reaction, which is more pronounced in some patients, increasing the postoperative recovery period and treatment cost [2].

Primary closure of the surgical wound is done by tight approximation of edges and this kind of procedure is routinely practiced and is quite conventional. Nonetheless, this type of healing can lead to considerable postoperative complications due to the inability of inflammatory exudate to escape out. Additionally, there are chances of primary closure undergoing dehiscence and leading to secondary healing [3]. An alternative option to this technique is secondary closure of wound by different methods, wherein the socket remains in communication with oral cavity by creating a pseudo-socket through a wedge preparation of mucosa about 5-6 mm distal to the second molar following closure of flap [4]. This type of wound closure allows the escape of inflammatory exudate from surgical site, thereby maintaining a self-irrigating opening [5]. There have been other ways of providing secondary healing by insertion of drains, gauze dressings and modified flap designs [6,7].

Against this backdrop, the current study was undertaken to assess and compare the primary and secondary wound closure techniques after removing impacted mandibular third molars and assessing the difference between primary and secondary surgical wound closure on postoperative pain, swelling and amount of mouth opening.

\section{Material and Methods}

\section{Study Design and Sample}

This study utilized a split-mouth design in which the patients served as their own controls and was conducted from May 2018 to December 2018.

A total of 30 participants ranging between 18-30 years of age and of either sex who had bilaterally impacted mandibular third molars were selected by systematic random sampling method from patients attending the Department of Oral and Maxillofacial Surgery, VK Institute of Dental Sciences, Karnataka, India.

Patients needing surgical extraction of impacted third molars and those who willingly participated and agreed for the postoperative follow-up were included in the study. The exclusion criteria considered were the patients with contradictions to the drugs used in the dental procedure and any comorbidity that would contraindicate minor oral surgical procedures.

Baseline Evaluation

Complete oral examination was done for the enrolled participants and the impacted mandibular third molars were diagnosed by established clinical and radiographic parameters (Figure 1). The severity of pain was evaluated using the visual analog scale (VAS). Participants were requested to gauge their pain on a scale of o10, where 0 specified no pain and 10 specified the most severe pain.

The selected participants were distributed randomly into the following two groups: Group 1: Primary wound closure after removal of left mandibular impacted third molar and Group 2: Secondary wound closure after removal of the right mandibular impacted third molar. 


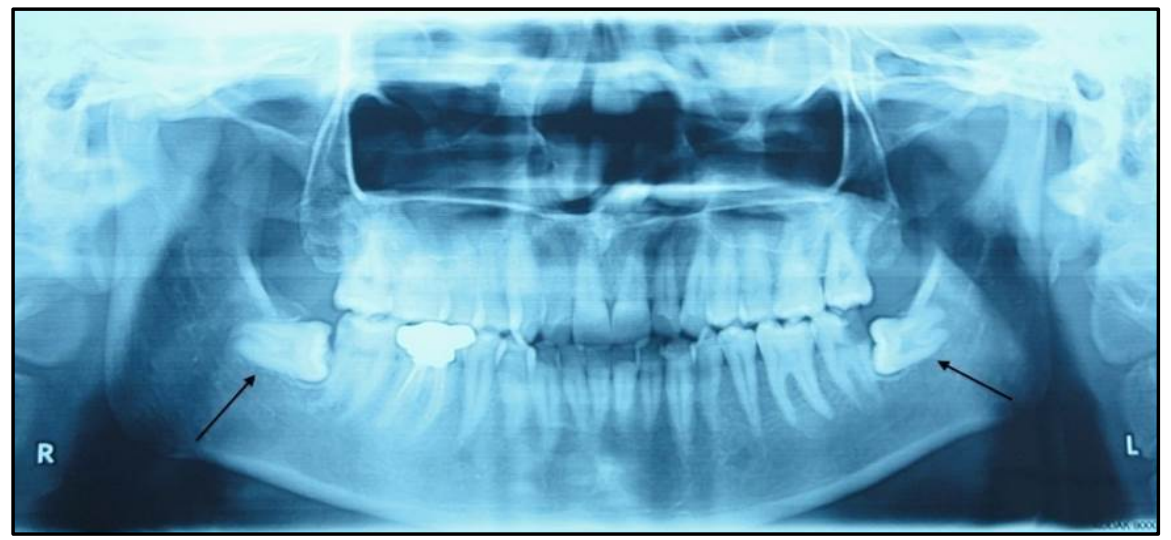

Figure 1. Orthopantomogram showing bilaterally impacted mandibular third molars.

Surgical Protocol

The position of impacted tooth was evaluated by utilizing Winter's [8] and Pell and Gregory's classifications [9]. All the selected participants underwent surgical extraction of the impacted mandibular third molar in the oral surgery unit by a single surgeon. Pre-operative antimicrobials, analgesics or other drugs that might affect the healing outcome were not given to the participants.

Anaesthesia was attained by blocking the inferior alveolar nerve, lingual nerve and long buccal nerve with $2 \%$ lignocaine with 1:80000 adrenaline concentration. A full-thickness incision was used to prepare a trapezoidal flap. After raising the flap, ostectomy and odontotomy were completed using burr on a straight handpiece under copious irrigation. After finishing the extraction, curettage of socket and irrigation were done with a $0.9 \%$ saline solution. In group 1, the flap was repositioned and sutured using 3-0 silk suture (Figure 2A), whereas, in group 2, a wedge of mucosa with a width of 5-6 mm that was distal to second molar was removed, followed by the repositioning and suturing of the flap (Figure $2 \mathrm{~B}$ ).

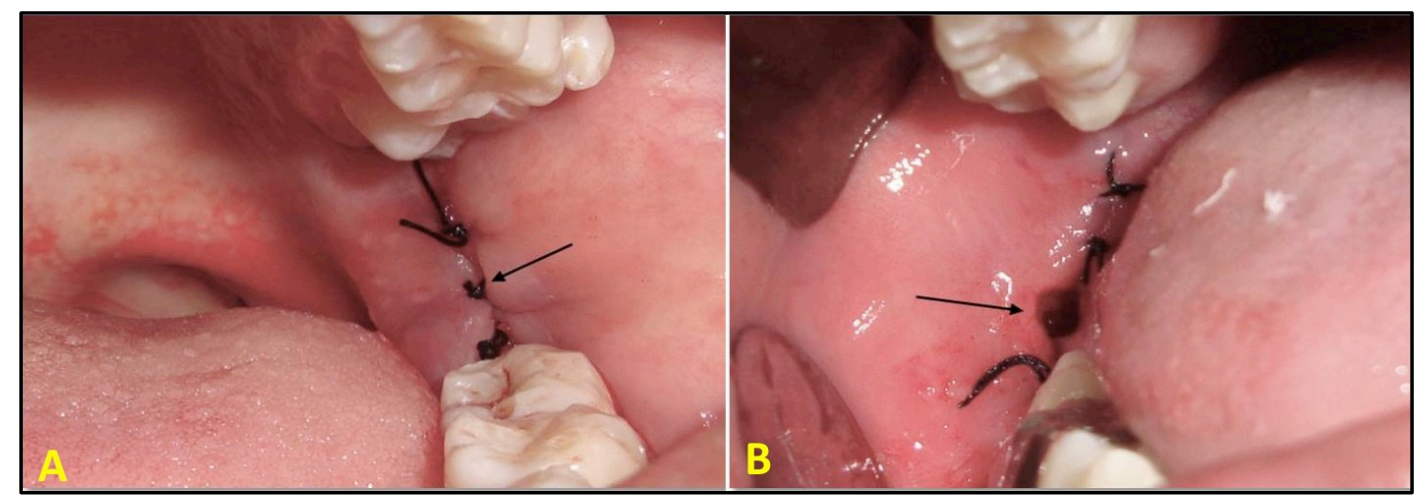

Figure 2. A) Group 1: extraction of left mandibular impacted third molar followed by primary closure. B) Group 2: extraction of right mandibular impacted third molar followed by secondary closure.

Subsequently, all the participants received postoperative instructions and medications, including antibiotics (amoxicillin $500 \mathrm{mg}$ thrice a day for 5 days) and analgesics (a combination of diclofenac and paracetamol twice a day for 3 days). Participants were also requested to maintain a record of pain and swelling during the subsequent 7 days.

Follow-up Evaluations 
Patients were recalled after 24 hours and on $7^{\text {th }}$ day for follow-up and sutures removal. During these calls, any variations in the VAS along with difficulty in eating, speech and mouth opening were duly noted on the proforma. Maximum mouth opening was measured between the incisal edges of maxillary and mandibular central incisors using a measuring tape to rule out trismus. Swelling was measured utilizing validated landmarks (angle-incisor distance and tragus-incisor distance) and evaluating the distance between them preand postoperatively. Also, the presence of any signs of other postoperative complications was duly noted. Patients were assessed and evaluated till $7^{\text {th }}$ day for postoperative complications in both the groups and compared.

Statistical Analysis

All data were tabulated and evaluated using SPSS software, version 17.0 (IBM Corp., Armonk, NY, USA). Mann-Whitney $\mathrm{U}$ test and Wilcoxon matched-pairs test were utilized for comparison between the groups for VAS. Comparison for swelling was attained by t-test. A p-value $\leq 0.05$ was assigned as statistically significant.

\section{Ethical Clearance}

The study received ethical approval from the institute (Protocol No. KLE/604), and the written informed consent was obtained from all the participants before starting the surgical procedure.

\section{Results}

In total, 30 patients (19 male and 11 female patients) consented to participate in the current study. The average age of the participants was 24.7 years. Statistically significant improvement in mouth opening in group 2 compared to group 1 was noticed, with a higher percentage of increased values from pre-operative to day $1(\mathrm{p}=0.0005)$ to day $7(\mathrm{p}=0.00001)$ in group 1 , suggestive of more trismus.

Comparing group 1 and group 2 concerning pain scores from day 1 to day 7 by Mann-Whitney U test and Wilcoxon matched-pairs test, there was no statistically significant difference between the two groups $(\mathrm{p}=0.98)$. However, on comparing the swelling among both the groups, there was statistically significant results $(\mathrm{p}=0.02)$ suggesting less swelling in the group 2 patients (Figures 3 and 4 ).

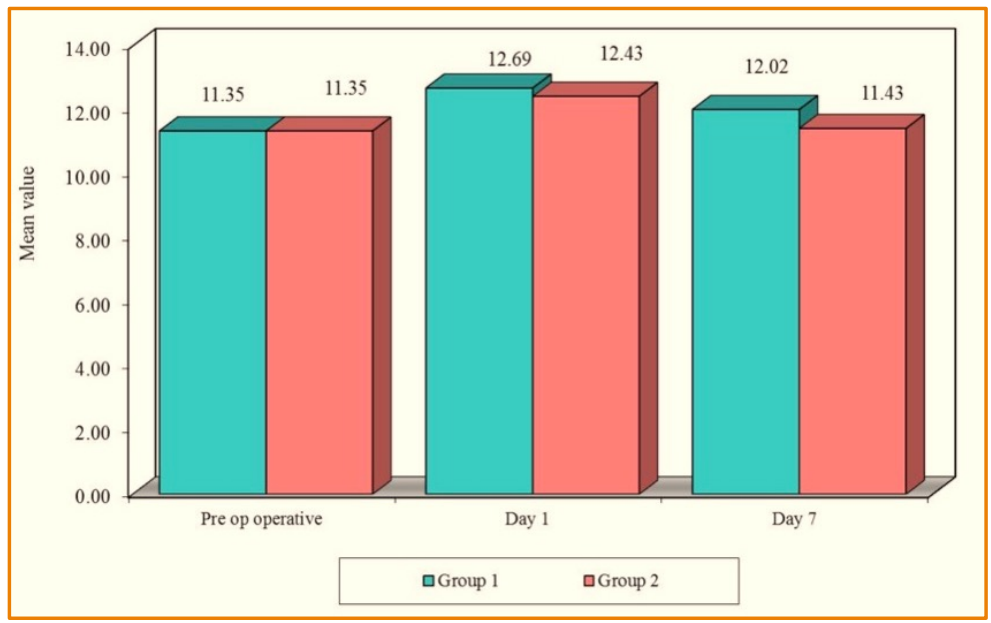

Figure 3. Comparison concerning angle-incisor distance at pre-operative, day 1 and day 7. 


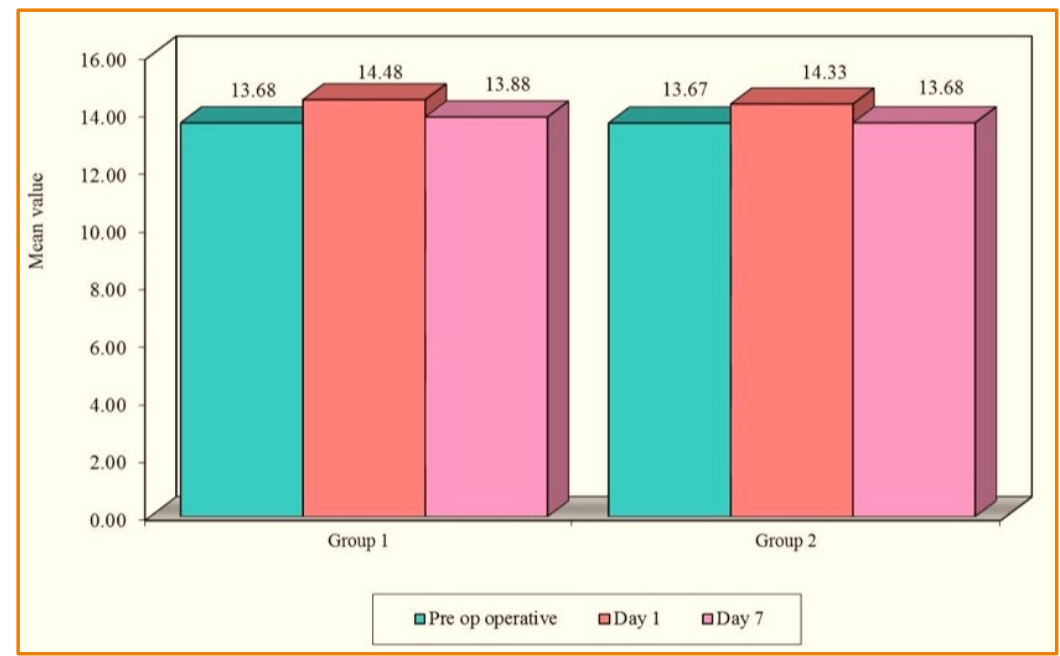

Figure 4. Comparison concerning tragus-incisor distance at pre-operative, day 1 and day 7.

\section{Discussion}

Surgical extraction of impacted teeth is a routinely practiced dental procedure that demands technical skills, thorough knowledge of anatomy and surgical principles, justification of antibiotic usage, effective anesthesia, appropriate drug prescription and total patient care. Like other surgical procedures, mandibular third molar surgical extraction is associated with postoperative complications like pain, swelling, infections, alveolar osteitis, and paresthesia [10]. These complications occur either due to the dental practitioner's carelessness during removal or due to the patient's failure to not follow the postoperative instructions properly $[11]$.

Since the severity of pain and swelling might vary among individuals, the risk of error is present when the case and control are different individuals. Hence to avoid this, a split-mouth study method was used in this study in which the participants served as their own controls. The extent of pain and/or swelling is the main symptom of the patient's comfort during the recovery period [12]. In this study, pain and swelling were subjectively evaluated by VAS, whereas an objective evaluation of swelling was done by utilizing validated landmarks. Many studies have also suggested photographic techniques and CT scanning to evaluate anatomical changes in the patients planned for third molar removal [13]. Swelling generally reaches its maximum size within 24 to 88 hours after the surgical procedure and begins to reduce on the $3^{\text {rd }}$ or $4^{\text {th }}$ day. It is usually subsided completely by the end of the $1^{\text {st }}$ week [14]. So the participants in the current study were followed up after 24 hours and on the $7^{\text {th }}$ day for evaluation. According to Berge TI, VAS is a consistent and repeatable method for assessing the patient's swelling [15]. Hence, in this study, patients were provided VAS for pain and swelling and were requested to record on the self-analysis proforma for 7 days. Validated extraoral anatomical landmarks were also measured and compared.

The factors determining the wound healing include host defense mechanism, type of healing, time of procedure, types of flaps, extent of reflection of the mucoperiosteal flap, necessity for tooth sectioning, bone removal, and skills surgeon [16]. In this study, all the parameters contributing to wound healing were consistent in the patients except the closure technique. Secondary closure technique which employed cutting a 5-6 mm wedge of mucosa distal to mandibular second molar followed by suturing of the flaps, allowed the escape of inflammatory exudate from the extraction site through the pseudo-socket created, thereby reducing pain and swelling. Hence, the current study results revealed that the secondary closure of the extraction site is 
convenient for the patient due to less post-extraction pain, swelling, and improved mouth opening. These findings are consistent with the study conducted by Pasqualini et al. [3] and Rakprasitkul and Pairuchvej [6].

Results of the current study showed that group 2 had reduced pain and swelling when calculated from day 1 to day 7 as compared to group 1, which is in agreement with the studies done by Dubois et al. [17] and Holland and Hindle [18] who postulated that secondary closure reduced pain and swelling in the immediate postoperative period and also reduced the discomfort of the patient.

In a comparison of the tragus-incisor distance between two groups, no statistically significant difference was found. The swelling was more prominent on the angle to incisor distance. The data received from VAS from the patients indicated that group 2 experienced less pain and swelling than group 1, thus favoring the secondary type of closure. On comparing the primary and secondary closures and assessing the primary and secondary healing, we found results stating that the complications like pain, swelling, and reduced mouth opening were markedly improved in the group with secondary closure technique than in primary closure.

\section{Conclusion}

After third molar extraction, the type of wound healing affects postoperative complications and predicts the level of discomfort that patients may experience in the postoperative recovery period. The secondary closure provides escape of inflammatory exudate from healing socket, thereby markedly reducing postoperative pain and swelling with better mouth opening than the primary healing. Thus, secondary closure of the third molar extraction wound provides superior postoperative results compared to healing by primary closure.

\section{Authors' Contributions}

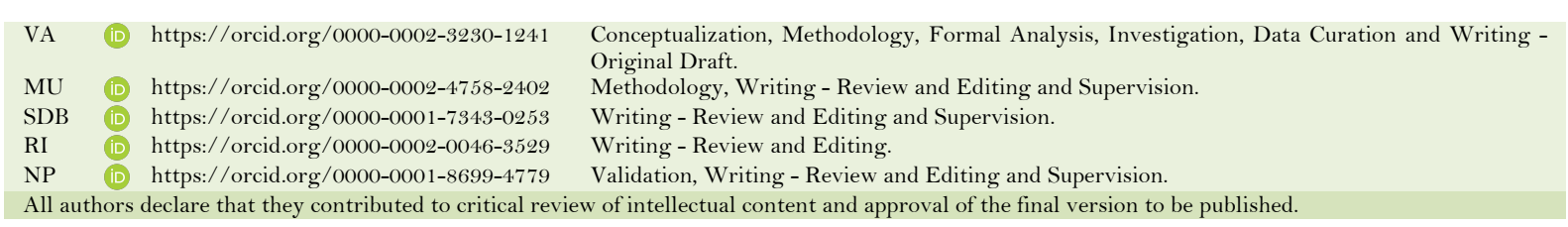

\section{Financial Support}

None.

\section{Conflict of Interest}

The authors declare no conflicts of interest.

\section{Data Availability}

The data used to support the findings of this study can be made available upon request to the corresponding author.

\section{References}

[1] Balakrishnan G, Narendar R, Kavin T, Venkataraman S, Gokulanathan S. Incidence of trismus in transalveolar extraction of lower third molar. J Pharm Bioallied Sci 2017; 9(Suppl 1):S222-S227. https://doi.org/10.4103/jpbs.JPBS_161_17

[2] Vallerand WP, Vallerand AH, Heft MJ. The effects of postoperative preparatory information on the clinical course following third molar extraction. Oral Maxillofac Surg 1994; 52(11):1 165-70. https://doi.org/10.1016/0278-2391(94)90536-3 
[3] Pasqualini D, Cocero N, Castella A, Mela L, Bracco P. Primary and secondary closure of the surgical wound after removal of impacted third molars: a comparative study. Int J Oral Maxillofac Surg 2005; 34(1):52-7. https://doi.org/10.1016/j.ijom.2004.01.023

[4] Khande K, Saluja H, Mahindra U. Primary and secondary closure of the surgical wound after removal of impacted mandibular third molars. J Maxillofac Oral Surg 2011; 10(2):112-7. https://doi.org/10.1007/s 12663-011-0216-y

[5] Al-Samman AA. A review of wound closure technique-patient morbidity relationship after wisdom tooth surgery. Iraqi Dent J 2016; 38(3):154-9. https://doi.org/10.26477/idj.v38i3.101

[6] Rakprasitkul S, Pairuchvej V. Mandibular third molar surgery with primary closure and tube drain. Int J Oral and Maxillofac Surg 1997; 26(3):187-90. https://doi.org/10.1016/s0901-5027(97)80817-x

[7] Dinah F, Adhikari A. Gauze packing of open surgical wounds: Empirical or evidence-based practice? Ann R Coll Surg Engl 2006; 88(1):33-6. https://doi.org/10.1308/003588406X83014

[8] Winter GB. Impacted Mandibular Third Molar. St. Louis: American Medical Book; 1926.

[9] Pell GJ, Gregory BT. Impacted mandibular third molars: Classification and impacted mandibular third molars: Classification and modified technique for removal. Dent Digest 1933; 39:330-8.

[10] Deliverska EG, Petkova M. Complications after extraction of impacted third molars - Literature review. J IMAB 2016; 22(3):1202-11. https://doi.org/10.5272/jimab.2016223.1202

[11] Alvira-Gonzalez J, Gay-Escoda C. Compliance of postoperative instructions following the surgical extraction of impacted lower third molars: a randomized clinical trial. Med Oral Patol Oral Cir Bucal 2015; 20(2):e224-e230. https://doi.org/10.4317/medoral.20121

[12] Wells N, Pasero C, McCaffery M. Improving the Quality of Care Through Pain Assessment and Management. In: Hughes RG, editor. Patient Safety and Quality: An Evidence-Based Handbook for Nurses. Rockville (MD): Agency for Healthcare Research and Quality (US); 2008. Chapter 17. Available from: https://www.ncbi.nlm.nih.gov/books/NBK2658/. [Accessed on July 20, 2020].

[13] Suddhasthira T, Chaiwat S, Sattapongsda P. The comparison study of primary and secondary closure technique after removal of impacted mandibular third molars. Thai J Oral Maxillofac Surg 1991; 5:67-73.

[14] Peterson LJ. Principles of Management of Impacted Teeth. In: Peterson LJ, Ellis E III, Hupp JR, Tucker MR, editors. Contemporary Oral and Maxillofacial Surgery. $4^{\text {th }}$.ed. St Louis: CV Mosby; 2003.

[15] Berge TI. Inability to work after surgical removal of mandibular third molars. Acta Odontol Scand 1997; 55(1):64-9. https://doi.org/10.3109/00016359709091944

[16] Pippi R. Post-surgical clinical monitoring of soft tissue wound healing in periodontal and implant surgery. Int J Med Sci 2017; 14(8):721-8. https://doi.org/10.7150/ijms.19727

[17] Dubois DD, Pizer ME, Chinnis RJ. Comparison of primary and secondary closure techniques after removal of impacted third molars. J Oral Maxillofac Surg 1982; 4O(10):631-4. https://doi.org/10.1016/0278-2391(82)90111-2

[18] Holland CS, Hindle MO. The influence of closure or dressing of third molar sockets on post-operative swelling and pain. Br J Oral Maxillofac Surg 1984; 22(1):65-71. https://doi.org/10.1016/0266-4356(84)90011-1 